\title{
Ovarian tumours- Incidence and distribution in a tertiary referral center in south India
}

\author{
Dr.M.Yogambal ${ }^{1}$, Dr. P. Arunalatha ${ }^{2}$, Dr.K.Chandramouleeswari ${ }^{3}$, \\ Dr.V.Palaniappan ${ }^{4}$ \\ ${ }^{1}$ (Department of pathology, Stanley medical college, The Tamilnadu Dr.M.G.R medical University, India) \\ ${ }^{2}$ (Department of pathology, Stanley medical college, The Tamilnadu Dr.M.G.R medical University, India) \\ 3 (Department of pathology, Stanley medical college, The Tamilnadu Dr.M.G.R medical University, India) \\ ${ }^{4}$ (Department of pathology, Stanley medical college, The Tamilnadu Dr.M.G.R medical University, India)
}

\begin{abstract}
Ovarian neoplasms have become increasingly important not only because of the large variety of neoplastic entities but more so because they have gradually increased the mortality rate due to female genital cancers. Diverse histopathologies are common in ovarian tumours which reflect its different cell of origin. A total of 7492 gynaecology cases were received at the Department of Pathology, Stanley Medical College, Chennai, India, during the period of January 2009 to December 2013. In this 402 ovarian tumours were studied to find out frequency of different histological patterns of ovarian tumors at our tertiary care institute. Among 402 ovarian tumour cases, majority 316 (78.6\%) were benign, but an alarming number of 83 (20.65\%) were malignant, remaining 3 cases were borderline. The commonest histological pattern observed in the study was epithelial tumors $(71.64 \%)$. The commonest benign tumours were serous cyst adenoma and teratoma, while; the commonest malignant tumors were serous cystadenocarcinoma and mucinous cystadenocarcinoma. Epithelial tumors were commonest variety of ovarian tumors followed by germ cell tumors. A relatively high number of malignancies were observed in this study.
\end{abstract}

Key words: Ovarian tumour, epithelial tumour, germ cell

\section{Introduction:}

In ovarian tumours diverse histopathologies are common reflecting the different cell origins of the tumours. The exact incidence in south India is not known but ovarian cancer is the one of the common cancer among females of India and continues to present at an advanced stage.[1] Japan and Asian countries have rates of 2-6.5 new cases per 100,000 women per year.[2] Ovarian carcinoma represents the sixth most common female cancer and the fourth leading cause of death due to cancers in women and is seen predominantly after $3^{\text {rd }}$ decade.[3] Ovarian cysts of benign nature may occur at any point in the life but they are most common during childbearing age and constitute about $90 \%$ of ovarian tumours.[4]

Most benign tumours are cystic and finding of solid elements make malignancy more likely. The most recent Surveillance, Epidemiology and End Results (SEER) calculations of lifetime risk for ovarian cancer are that 1 in 55 women will develop ovar ian cancer over their lifetime[5] Ovarian tumours are generally difficult to detect until they are advanced in stage or size, as the symptoms are vague and manifest over time.

Determination of various histologic patterns of ovarian tumours is very important in diagnosis, prognosis as well as treatment of ovarian tumours. Prognosis of the tumours can also be predicted from the degree of differentiation of the tumours. Primary tumours are classified into surface epithelial tumours, germ cell tumours, sex cord stromal tumours, germ cell sex cord stromal tumours, tumours of rete ovarii and miscellaneous tumours of which surface epithelial tumours are most common. The stage and laterality of the tumour also indicates their nature for example, tumours in the sex cord stromal category are almost always confined to a single ovary. On the other hand, approximately $65 \%$ of the metastatic tumours are bilateral. In this study we have analysed frequency of ovarian tumours and histopathological patterns in our tertiary care hospital.

\section{Materials And Methods:}

This study was a retrospective and descriptive study done for a period starting from January2009 to December 2013(5 years) at the Department of Pathology, Stanley Medical College, Chennai, India. All cases diagnosed as ovarian tumours both primary (Benign and malignant) and metastatic were included in the study. 
The diagnosis of ovarian tumour is based on histopathology conducted in our pathology department. This was a descriptive study describing the frequency of benign and malignant ovarian tumours in our tertiary care institute along with age of presentation and histopathological pattern. Patients with abdominopelvic masses supported by clinical, transvaginal USG/CT/MRI presenting as primary ovarian masses were included in this study. Ovarian masses with GIT lesions, endometrial carcinoma, cervical carcinoma were excluded from the study.The acquired data were analyzed using the descriptive statistics. Frequency of the ovarian tumours (benign and malignant) was determined and the age of presentation of ovarian tumours were analysed.

\section{Results:}

Total numbers of gynaecological cases during the study period were 7492. Total numbers of ovarian tumours studied in this five year period were 402. Out of which benign ovarian tumours were 316 (78.6\%)(Figure-1) and malignant ovarian tumours were 83 (20.65\%) Border line tumours were 3(0.75\%). The commonest histological patterns observed in the study were epithelial tumours $(71.64 \%)$ including both benign and malignant epithelial tumours. The frequency of different histopathological types of benign ovarian tumour showed that the commonest tumour was serous cyst adenoma $(21.4 \%)$ followed by mature c ystic teratoma (19.9\%) (Figure-2 \&3) Common malignant ovarian tumours were serous cyst adeno carcinoma $(9.5 \%)$ (Figure 4) and mucinous cyst adeno carcinoma (3.2\%) (Figure-5) the commonest presenting symptom was pain abdomen followed by mass abdomen (Table-1). The incidence of ovarian tumour presenting at secondary is 3 cases $(0.75 \%)$ (Figure 6$)$.

\section{Discussion:}

Our study reveals that the Presentation of the ovarian tumour is variable. Some of the ovarian tumours may be incidentally diagnosed on ultrasound whereas others may present with acute abdominal pain. In our study the commonest presenting symptom was pain abdomen 269(66.92\%)followed by mass abdomen 113(28.11\%).The results comply well with a study carried out at by Rashid et al.[6] in which abdominal pain was the commonest presenting complaint (59\%) followed by abdominal mass/distension (37\%). In contrast to another retrospective analysis by Jamal et al [7] the commonest mode of presentation was bleeding per vaginum, followed by pain abdomen, pelvic mass and gastric intestinal symptoms. The exact nature of all ovarian tumour cannot be confirmed preoperatively just by clinical examination. Australian ovarian cancer research program supports CA 125 serum evaluation in addition to pelvic examination, transvaginal ultrasonography for early detection and screening of ovarian tumours. MRI and CT with image guided biopsies may also be furthersupportive [8].The microscopic appearance of the tumour is a must to find the histopathological pattern upon which further management rests. The grading of tumour type also has its significance in further management.

Among histopathologic types, the commonest category of the ovarian tumours encountered in our series was epithelial tumour followed by germ cell tumours (Figure 7). The most common benign tumour was serous cyst adenoma 86 cases $(21.4 \%)$ followed by mature cystic teratoma. Serous cyst adenomas were shown the commonest among the Indians and teratoma were the commonest benign tumour among the Malays and Chinese [9]. Serous tumours were found to be more common than mucinous. Similar results were reported by Prabhakar et al in which serous tumours were the commonest followed by mucinous tumours [10]. Some molecular and histological evidence suggests that mucinous epithelial ovarian cancers develop via a sequence from benign tumour through borderline tumour to invasive cancer which suggests the potential preventability of borderline and invasive mucinous ovarian cancer by surgical excision of identifiable precursor lesions.[11] A retrospective study by Ahmed et al showed benign cystic teratoma to be the commonest benign tumour(35.17\%)[12]

The study in Belgium by Pilli et al explored epithelial tumours to be the commonest variety constituting $70.9 \%$ of all the ovarian tumours. Second most common to be the germ cell tumour $(21.2 \%)$ followed by sex cord stromal tumours $(6.7 \%)$ and metastatic tumours $(0.7 \%)[13]$

In our study most common tumour in adolescent and adult age group (10-30 years) is germ cell tumour constitute about 3.23\%. These results are similar to Mencezer et al study [14]. In this study the incidence rate for the total group of ovarian malignancies in the 0-19 age group was 0.52 and for ages 5-19 it was 0.71 per 100,000.Most common tumours among middle age and older age group are surface epithelial tumours. This is in correlation with most of the western literature.

Tumours in the borderline category are characterized by epithelial proliferation greater than that of the benign tumour more than two layers and less than four layers stratification but an absence of destructive invasion of the stroma [15]. .In our series of patient we encountered 3 cases of ovarian tumour with borderline malignancy. In this 2 cases were atypical proliferative mucinous tumour and one was atypical serous proliferating tumour. Among the non-neoplastic cysts follicular cysts were the commonest 
(31.34\%). Follicular cysts and corpus luteal cysts were the commonest $(80.2 \%)$ non-neoplastic lesions in a study by Gupta.[16]. Common malignant ovarian tumours were serous cyst adeno carcinoma (9.5\%) and mucinous cyst adeno carcinoma (3.2\%). An important aspect regarding histological pattern of malignant tumours is the degree of differentiation depicting the grade of tumour. The staging as per FIGO which is strictly adhered for clinical management (Table -2).The 5 year survival is associated with both stage and grade of disease. The 5 year survival rates for tumour stage I to IV are $85 \%, 71 \%, 41 \%$ and $22 \%$ respectively the survival rates with poorly differentiated tumours are significantly worse than with moderate or well differentiated tumours. There are several genetic risk factors that predispose to ovarian cancers. They are BRCA1, BRCA2 in which breast cancer associated with ovarian cancer, and HNPCC in which colorectal carcinoma associated with ovarian cancer. Smoking, obesity and eating high fat diet are some of the predisposing factors for ovarian cancer. Women who have not had children, are unable to have children, have never used oral contraceptives or have had children over the age of 30, may be slightly more at risk. Early puberty (menstruating before12) or late menopause (onset after 50) is also risk factor. Other non conclusive risk factors are women who are using oestrogen only hormone replacement therapy and exposure to multiple fertility drugs[8]. Finally we have included Grossing of ovarian specimen in methodical way. Grossing of ovarian specimen:

The most common ovarian specimens encountered in surgical pathology are oophorectomies (with or without hysterectomy) and cystectomies. The weight and gross measurements of all three dimensions should be recorded. The laterality of tumour should be identified. The capsule should be inspected for areas of rupture, adhesions, tumor involvement, or other lesions. The ovary should then be bivalved along the long axis, and any lesions on the cut surface should be noted. Solid, cystic, and papillary lesions should be thoroughly sampled (one section per $\mathrm{cm}$ ). If no lesions are identified, one section for every $2 \mathrm{~cm}$ is adequate. If cysts are present, the colour and consistency of the cyst fluid should be noted, and any areas of nodularity or papillary excrescences should be sampled. Prophylactic oophorectomy specimens from excisions performed for a family history of a hereditary cancer syndrome should be cut perpendicular to the long axis and entirely submitted.

The surgical management of malignant primary ovarian tumors includes a staging procedure, so an ovary excised for a primary malignancy will usually be accompanied by multiple abdominal peritoneal biopsies, omentectomy, and regional lymph nodes. The small peritoneal biopsies are submitted entirely. The omentum sample must be serially sectioned. If grossly visible tumor is present, only one section need be submitted; when no tumor is identified, at least five sections should be submitted. All identified lymph nodes should be submitted from lymph node dissections. [17]

\section{Conclusion:}

Epithelial tumours of ovary are the commonest ovarian tumour which is similar to the Western \& local data from other medical institutes. Germ cell tumours were next to epithelial ovarian tumours which are more common in adult and adolescent age group. Amongst malignant ovarian tumours late reporting is common and patients usually present in advanced stages of the disease. The histological type of ovarian tumor correlates with the prognosis of the tumour. Serum CA-125 screening along with annual pelvic examination after 35 years of age in women along with Transvaginal USG can be used as regular screening methods to evaluate early detection of ovarian cancer.

\section{References:}

[1] Parveen S, Ilyas N, Asghar S. Patterns of care for ovarian cancer: Patients at Institute of Nuclear Medicine and Oncology (INMOL) Lahore. Specialist J Pak Med. Sci 1999;15:209-15

2] Murad A. Ovulation induction and ovarian tumours: the debate continues. J Pak Med Assoc 1998;48:353-6.

[3] TortoleroL, Mitchell FM, Rhodes HE. Epidemiology and screening of ovarian cancer. Obstet Gynaecol Clin North Am 1994;21:63-75.

[4] Day N.E, Krishnan E. Epidemiology of gynaecological cancers. Gynaecology by Shaw R W. 2nd ed. Edinburgh: Churchill Living Stone, 1997; p. 477-87.

[5] Piver MS. Prophylactic Oophorectomy: Reducing the U.S. Death Rate from Epithelial Ovarian Cancer. A Continuing Debate. Oncologist 1996;1:326-30

[6] Rashid S, Sarwar G, Ali A. A clinicopathological Study of ovarian cancer. Departments of Radiotherapy and oncology Sir Ganga Ram Hospital and Mayo Hospital Lahore. J Pak Med Assoc 1998;36;117-25.

[7] Jamal S, Quddusi H, Mehmood A. A Clinico Histopathological analysis of 110 ovarian tumours. Pak J Med Sci 1997;14:1923.

[8] http://www.ovariancancer.net.au

[9] Thanikasalam K, Ho CM, Adeed N, Shahidan MN, Azizah WK. LinksPattern of ovarian tumours among Malaysian women at General Hospital, Kuala Lumpur. Med J Malaysia 1992;47:139-46.

[10] Prabarker, Maingi K. Ovarian tumours--prevalence in Punjab. Indian J pathol Microbiol 1989;32:276-81.

[11] Jordan SJ, Green AC, Whiteman DC, Webb P M. Australian Ovarian Cancer Study Group. Risk factors for benign, borderline and invasive mucinous ovarian tumors: epidemiological evidence of a neoplastic continuum? Gynecol Oncol 2007;107:223-30.

[12] Ahmed Z, Kiyani N, Hasan S. H, Muzaffar S. Gill M. S. Histological Patterns of ovarian neoplasia. J Pak Med Assoc 
2000;50:416-9.

[13] Pilli GS, Suneeta KP, Dhaded AV, Yenni VV. Ovarian tumours: a study of 282 cases: J Indian Med Assoc 2002;100:420, 423-4

[14] Menczer J, Sadetzki S, Murad H, Barda G, Andreev H, Barchana M.Childhood and adolescent ovarian malignant tumors in Israel. A nationwide study: Acta Obstet Gynecol Scand. 1999 Oct;78(9):813-7.

[15] Barakat R.R. Borderline tumours of the ovary. Obstet Gynaecol Clin North Am 1994;21:93-105.

[16] Gupta N, Bisht D, Agarwal AK, Sharma VK. Retrospective and prospective study of ovarian tumours and tumour-like lesions: Indian J Pathol Microbiol. 2007;50(3):525-7.

[17] Gross Examination and Tissue Sampling. In:Humphrey, Peter A.; Dehner, Louis P.; Pfeifer, John D., editors. The Washington manual of surgical pathology. $1^{\text {st }}$ ed.Newyork. Lippincott Williams \& Wilkins; 2008. P. 399.

Tables

Table-1: Clinical presentation of the cases $(n=402)$

Symptoms Cases Percentage

Pain abdomen $\quad 269 \quad 66.92$

Mass Abdomen $113 \quad 28.11$

Menstrual disturbance $11 \quad 2.74$

GIT disturbance 5

Urinary 4

Table 2-The FIGO staging for primary carcinoma of the ovary

\author{
Stage Description \\ I Growth limited to the ovaries \\ Growth limited to one ovary; no ascites present containing malignant cells. No tumor on the external surface; \\ IA \\ IB IC* \\ capsule intact. \\ Growth limited to both ovaries; no ascites present containing malignant cells. No tumor on the external \\ surfaces; capsules intact.
}

Tumor either stage Ia or Ib, but with tumor on surface of one or both ovaries, or with capsule ruptured, or with ascites present containing malignant cells, or with positive peritoneal washings.

\title{
II Growth involving one or both ovaries with pelvic extension. \\ IIA Extension or metastases to the uterus or tubes. \\ IIB Extension to other pelvic tissues.
}

Tumor either stage IIa or IIb, but with tumor on surface of one or both ovaries, or with capsule(s) ruptured, or 
IIC*

III

with ascites present containing malignant cells, or with positive peritoneal

washings.

Tumor involving one or both ovaries with histologically confirmed peritoneal implants outside the pelvis or positive regional lymph nodes. Superficial liver metastases equals stage III.

Tumor is limited to the true pelvis, but with histologically proven malignant extension to small bowel or omentum.

IIIA Tumor grossly limited to the true pelvis, with negative nodes, but with histologically confirmed microscopic

seeding of abdominal peritoneal surfaces, or histologic proven extension to small bowel or mesentery.

IIIB Tumor of one or both ovaries with histologically confirmed implants, peritoneal metastasis of abdominal

peritoneal surfaces, none exceeding $2 \mathrm{~cm}$ in diameter; nodes are negative.

IIIC Peritoneal metastasis beyond the pelvis $>2 \mathrm{~cm}$ in diameter or positive regional lymph nodes.

IV Growth involving one or both ovaries with distant metastases. If pleural effusion is present, there must be

positive cytology to allot a case to stage IV. Parenchymal liver metastasis equals

stage IV.

Data from FIGO Committee on Gynecologic Oncology. Current FIGO staging for cancer of the vagina, fallopian tube, ovary, and gestational trophoblastic neoplasia.

Int J Gynaecolobstet. 2009;105:3-4.

*To evaluate the impact on prognosis of the different criteria for allotting cases to stage Ic or IIc, it would be of value to know if rupture of the capsule was spontaneous or caused by the surgeon, and if the source of malignant cells detected was peritoneal washings or ascites.

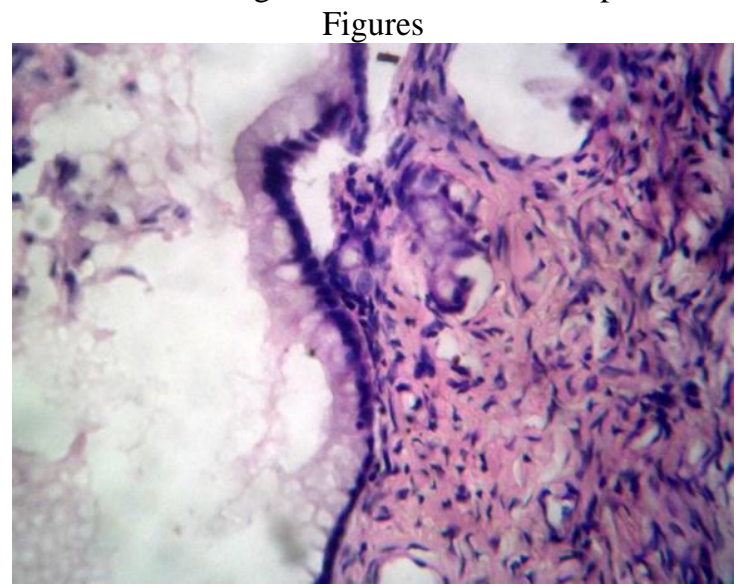

Figure 1: Mucinous cystadenoma of ovary

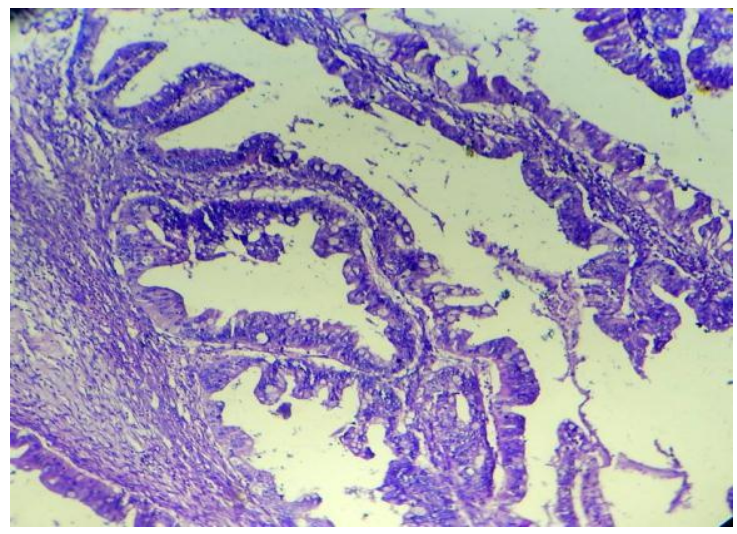

Figure 2: Atypical proliferating mucinous tumour 


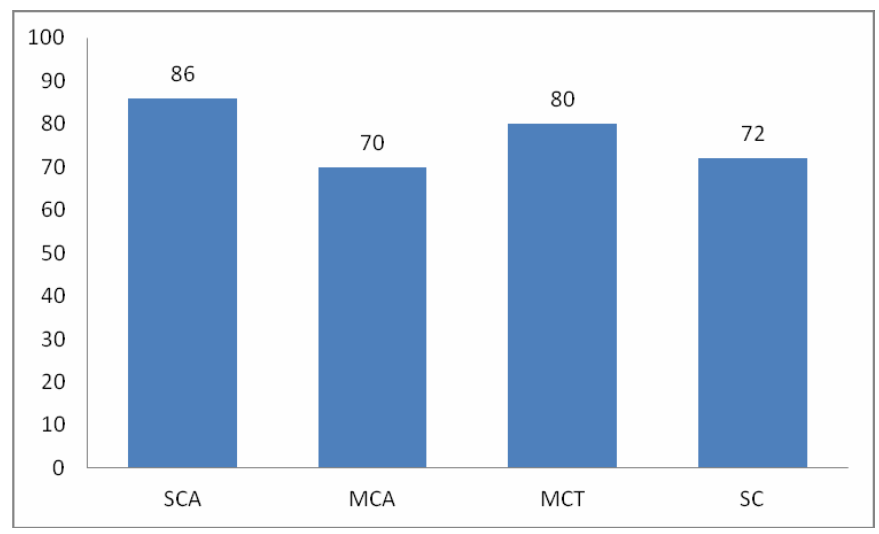

Figure-3: Frequency of Various histological types of Benign ovarian tumours (n=316) SCA= Serous cystadenoma, $\mathrm{MCT}=$ Mature cystic teratoma, $\mathrm{SC}=$ Serous cyst, $\mathrm{MCA}=$ Mucinous cyst adenoma

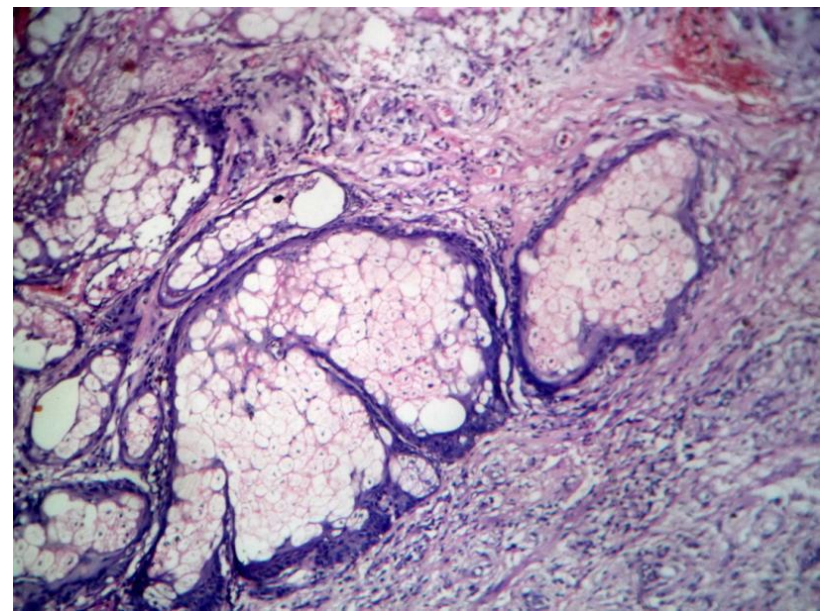

Figure 4: Mature cystic teratoma

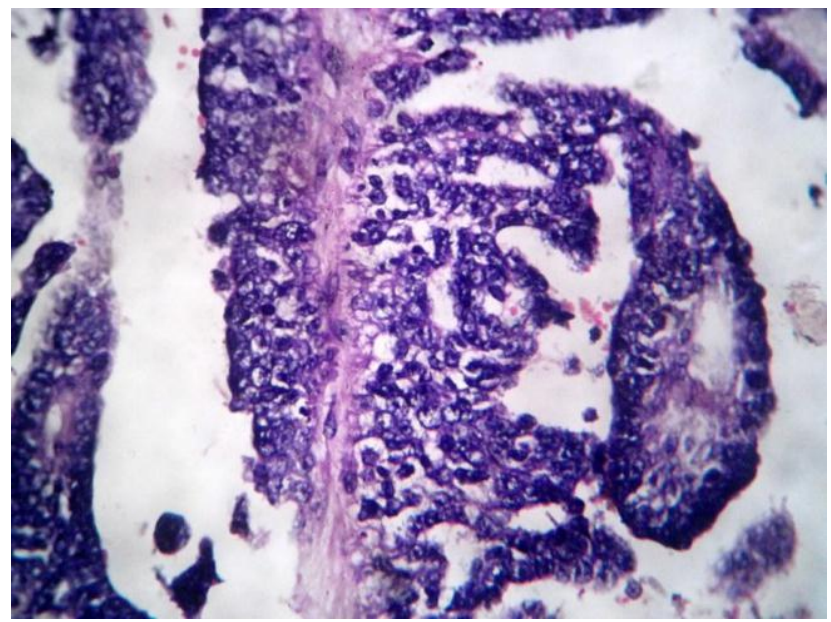

Figure 5: Serous cystadenocarcinoma 


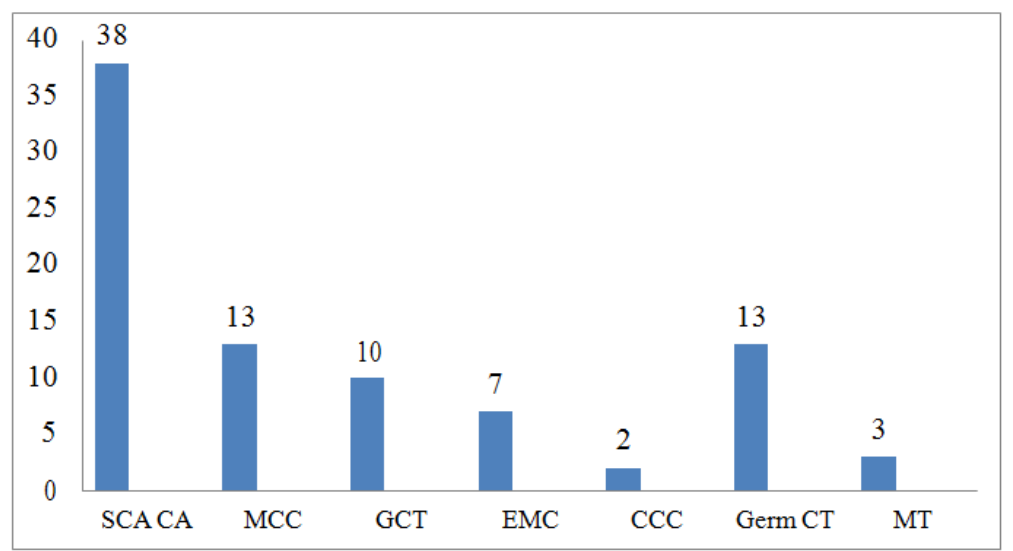

Figure-6: Frequency of various histological types of malignant ovarian tumours $(\mathrm{n}=83), \mathrm{SCA} C A=\operatorname{Serous}$ cyst adeno carcinoma, MCC=Mucinous Cystadeno Carcinoma ,

GCT $=$ Granulosa cell tumors, EMC=Endometriod Carcinoma, $\mathrm{CCC}=$ Clear cell Carcinoma Germ CT= Germ cell tumour, MT- Metastasis

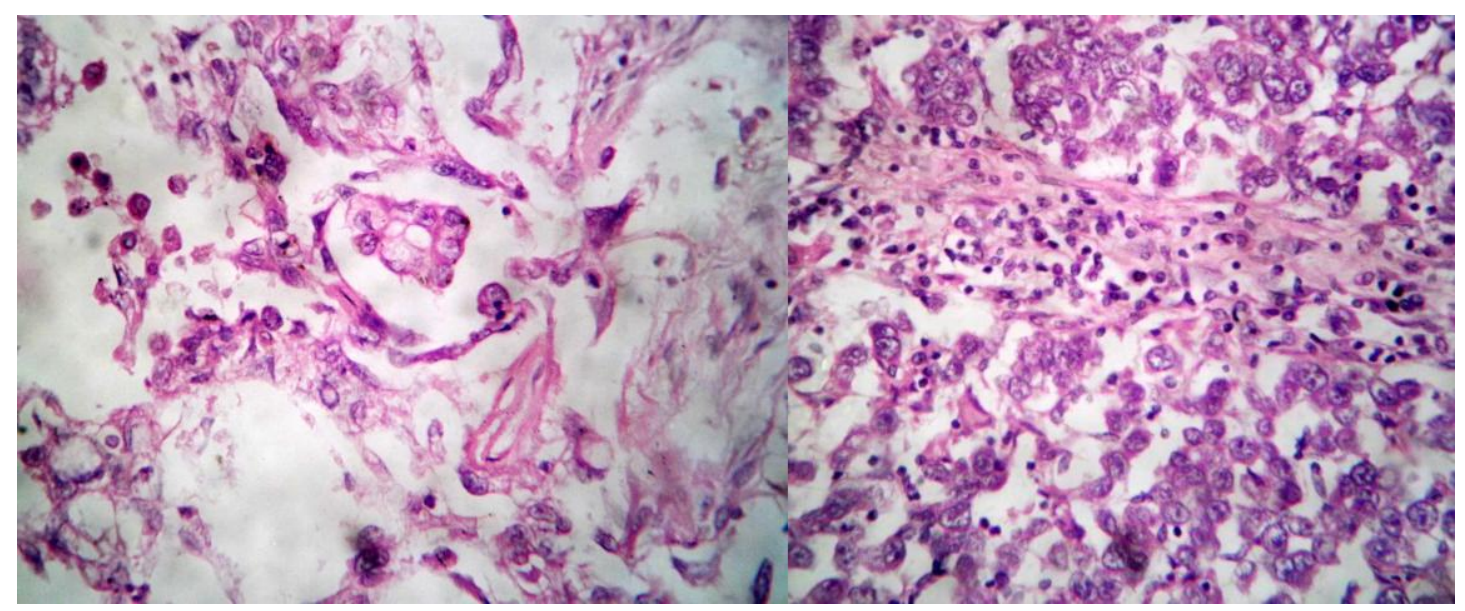

Figure captions \& legends:

Figure 7: Mixed germ cell tumour

Figure 1: Mucinous cystadenoma of ovary

Figure 2: Atypical proliferating mucinous tumour

Figure-3: Frequency of Various histological types of Benign ovarian tumours

Figure 4: Mature cystic teratoma

Figure 5: Serous cystadenocarcinoma

Figure-6: Frequency of various histological types of malignant ovarian tumours

Figure 7: Mixed germ cell tumour 